\title{
Forming of micro-components by electrical-field activated sintering
}

\author{
Jie Zhao a, Yi Qin, Kunlan Huang, Muhammad Bin Zulkipli, and Hasan Hijji \\ Centre for Precision Manufacturing, Department of Design, Manufacture and Engineering \\ Management, University of Strathclyde, 75 Montrose Street, Glasgow G1 1XJ, UK
}

\begin{abstract}
Recent research work has been undertaken to investigate the feasibility of forming micro-components by combining Electrical-field activated sintering and microforming (Micro-FAST). This paper firstly introduces the Micro-Fast technology and experimental validation method employed. Cylindrical components were used for the experiments and the sintering and forming was realised by use of a Gleeble 3800 thermalmechanical simulator. Thirteen different types of powders (metallic and ceramic) with variable particle sizes have been formed successfully. The influential parameters, such as pressure, temperature and heating rate, were studied. From the experiment results it is shown that the component quality depends significantly on the pressure, the heating rate and maximum temperature applied. Compared to other sintering technologies, the relatively short forming-cycle time of Micro-Fast (increased heating rate and reduced holding time) makes a good contribution to highly efficient particulate sintering for micro-manufacturing.
\end{abstract}

\section{Introduction}

At the present time, the large demands on the micro- or miniature-components have boosted the development of non-traditional manufacturing technologies with improved productivity and economic effectiveness [1]. Among these technologies, Electric Current Activated/Assisted Sintering (ECAS) has drawn ever growing attention for the sintering of particulate materials [2]. ECAS is a general term for a class of consolidation methods which adopt the combination of external electric field/currents with mechanical pressure for powder sintering, with enhanced interparticle bonding and densification. The invention of ECAS is dates from 1906, when Bloxam registered a patent on the sintering of powders using direct current in vacuum [3]. However, subsequent achievements of ECAS were very rare until the last two decades. From 1990, dramatic research interests and industrial applications have been reported from Japan, China, USA and from many other countries worldwide. Numerous papers have been published on fundamental research into process principles; whilst on the other hand, efforts have been made on apparatus development for industrial-scale production. Various technologies, such as, Pulsed Electric Current Sintering (PECS), Plasma Assisted Sintering (PAS), and Spark Plasma Sintering (SPS), have been invented and patented.

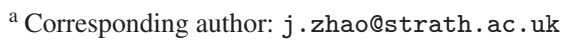

This is an Open Access article distributed under the terms of the Creative Commons Attribution License 4.0, which permits unrestricted use, distribution, and reproduction in any medium, provided the original work is properly cited. 

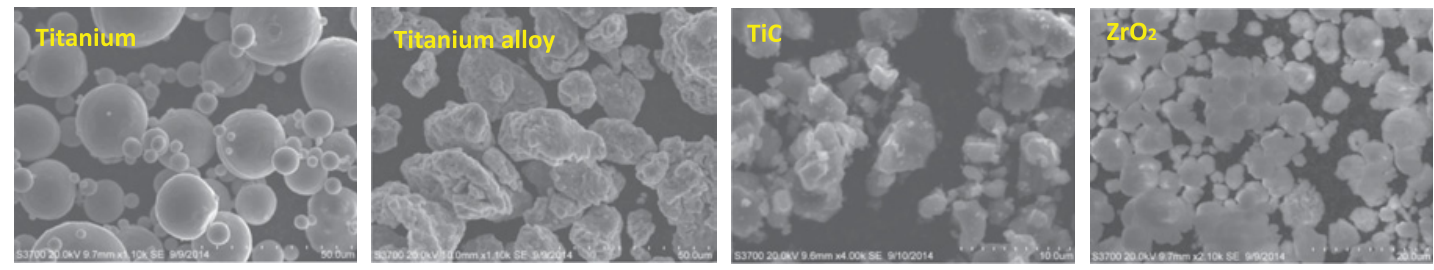

Figure 1. SEM Morphology of various powders.

It is worth drawing attention to electric current heating falling mainly into two categories: Resistance Sintering (RS) and Electric Discharge Sintering (EDS), where the former contains a low-voltage (a few tens of volts) and a high current (thousands of amps) with a characteristic waveform (direct current (DC), alternate current (AC), rectified current (RC), pulsed, etc.); whilst the latter relies on electrical energy suddenly discharged from a capacitor bank through a column of the workpiece powder contained within an electrically non-conducting tube [4]. Comparing with conventional sintering methods, such as pressureless sintering or hot pressing, the ECAS process has many advantages, includinga faster heating rate, a lower sintering temperature, a shorter holding time, the consolidation of difficult-tosinter-powders, the elimination of the need of sintering aids, no need of cold compaction, less sensitivity to the characteristics of the initial powders, and marked comparative improvements in the properties of the consolidated materials.

Despite SPS being one of the most recognised and widely applied processes, and having been approved for the forming of many types of materials, it has limits and drawbacks in respect of its lesser heating rate and that the pressure is influential on the final result during sintering. In this research paper, the focus is on the application of FAST (Field-activated Sintering Technology) to the forming of micro-components where FAST shows particular merits since small volumes of materials are to be heated up, such as ultra-fast heating and cooling rate (and hence, maintaining nano-structures of powder is possible), large plastic deformation of particles to increase density of the parts formed (different sintering mechanism, comparing to that for sintering of large-size parts), etc. The main differences between this process with SPS and others are: AC current applied for high heating efficiency; larger heating rate-, holding time- and pressure-dependent densification; and moreover, simplified process setup and control. Encouraging findings have been made using Micro-FAST with metallic materials $[5,6]$; however, the feasibility of forming ceramic materials still needs to be investigated further.

\section{Experimental procedure}

\subsection{Raw materials}

13 types of powders were used in the experiments. The initial materials are mainly classified into two material groups - metallic and ceramic. The powders are produced using different techniques, such as High Speed Ball Milling or Atomization, with the average particle size lying between 5-50 micros. The metallic materials mostly have a melting point of less than $1600^{\circ} \mathrm{C}$; however for the ceramic materials, the melting points can be as high as $3000^{\circ} \mathrm{C}$. The original powders were observed under SEM. The morphology of typical powers is shown in Fig. 1. Unlike conventional powder metallurgy, in which the powder is usually mixed with binder or additive, in the Micro-FAST process pure powder is applied directly for powder sintering. By doing this, the binder-removal process has been excluded and the final parts remain of high purity after a short sintering time. 

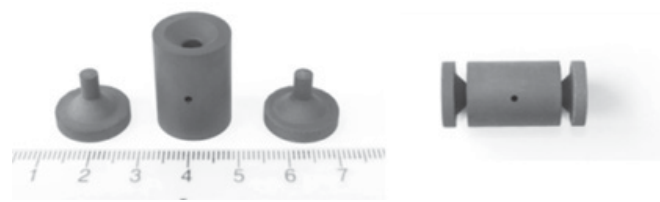

Figure 2. Graphite Punch and Die set.

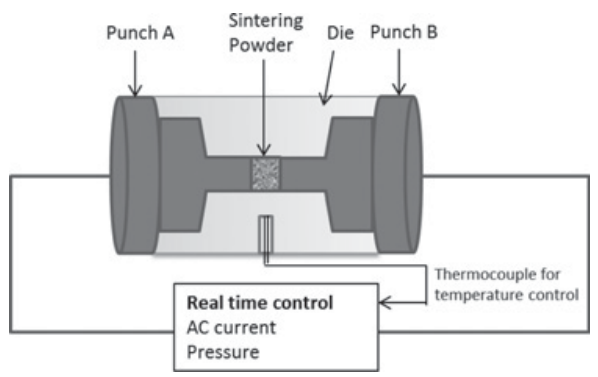

Figure 3. Illustration of the basic set up for the Micro-FAST experiment.

\subsection{Die-Set and other equipment}

As shown in Fig. 2, cylindrical die and punch set was designed and manufactured for the experiments to verify the material formability and to optimise the processing parameters. A small hole was drilled in the middle section of the die, in order to insert a thermocouple for temperature measurement. It is worth mentioning that several design changes were made to improve the overall performance of the die-set and to extend die life, which includes optimising die and punch geometry to reduce the heating time, optimisation of the punch shape to avoid thermal-stress concentration, and making the die-set symmetrical for better current conduction and uniform temperature distribution. For these experiments, all items of the die and punch were made from graphite.

The other equipment employed in the experiment was the Gleeble 3800 - a fully integrated digital closed-loop control thermal and mechanical testing system made by Dynamic Systems, which makes highly-accurate process control possible.

\subsection{The process principle}

The target samples to be formed were designed as $4 \mathrm{~mm}$ diameter and $4 \mathrm{~mm}$ height solid cylinders. The loose powders were weighted using a precision electric balance according to the calculated value (see Eq. (1)) and directly filled into the die cavity.

$$
m=\rho x V .
$$

As shown in Fig. 3, the closed die and punch set with filled powder was then placed horizontally between two electrodes on the Gleeble 3800. The pre-designed process parameters such as pressure, heating rate, temperature and time are input through a computer-based interface employing QuikSim Software. Upon starting the program, a constant uniaxial pressure was applied on the powders by pressing the punches, while the powders and die were heated dramatically by a high density AC current passing through the powder and/or the dies until the desired temperature was reached. The thermocouple was used for measuring the real-time temperature and providing feedback to the computer system; and in consequence, the input current or voltage was adjusted by an automatic controller to ensure that 


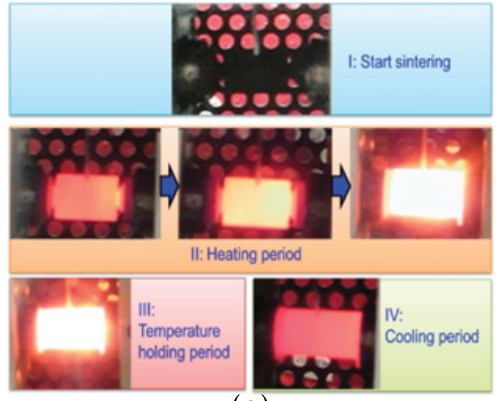

(a)

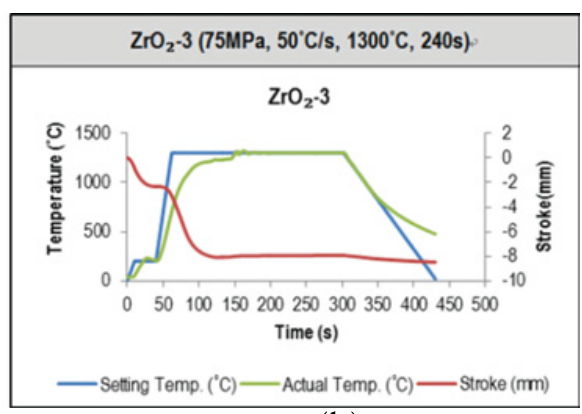

(b)

Figure 4. Processing sequence (a) and parameter curves (b) for $\mathrm{ZrO}_{2}$ during the sintering experiments.

the prescribed heating rate and temperature cycles were followed. The temperature and pressure were maintained for a given period of time, in order to consolidate the powder to a high density solid part. Thereby micro-forming was achieved under the joint action of particles boundary fusion and plastic deformation.

\subsection{Sintering experiments and parameters}

The experiments were conducted with variation of different key parameters, such as pressure, heating rate, maximum temperature and holding time. For the metallic powders with lower melting point and good electric conductivity, the sintering temperatures applied were less than $1100{ }^{\circ} \mathrm{C}$ with a heating rate of $100^{\circ} \mathrm{C} / \mathrm{s}$; while, for the ceramic materials with very high melting-point and low electric conductivity, the setting sintered temperature was as high as $1300^{\circ} \mathrm{C}$ with a heating rate of $50^{\circ} \mathrm{C} / \mathrm{s}$. In addition, all experiments were conducted within a vacuum environment to avoid the oxidation of the powder under high temperature.

For high-heating-rate sintering, a uniform temperature distribution becomes more crucial for forming fully homogeneous and dense compacts. Figure 4(a) shows the step-by-step sintering process of $\mathrm{ZrO}_{2}$ powder. At the beginning of the sintering, there was a gap between the punch and die. The gap reduced gradually during the heating period as the temperature increased. It can be noted that the gap disappeared during the temperature holding period which indicates an ideal current flow. It is worth mentioning that the temperature distribution shows a high uniformity during the whole sintering process irrespective of whether or not the gap disappeared. Figure 4(b) shows the corresponding time-dependent stroke and sintering temperature were obtained with the Gleeble output setup. The graph shows the setting temperature, the actual temperature and the stroke versus the time during the forming process. In terms of temperature control, it is seen from the curves that the actual temperature almost matches the setting temperature with only a small delay, from the beginning of the process. Once the set temperature is reached, the actual temperature remains stable without any deviations. Further, corresponding with the applied pressure, the stroke curve reduces continuously: good material flow can be observed as soon as the force is applied; a very steep drop occurring when the maximum temperature is achieved. Subsequently, during the holding time, the stroke has no obvious changes, which means that the powder compact can be accomplished very quickly after the powder reaches maximum temperature. However, the stroke changes during cooling stage, due to the shrinkage of the sintered part. Comparing with the other sintering methods for ceramic powders [4], seven minutes entire Micro-fast sintering cycle with maximum sintering temperature of $1300^{\circ} \mathrm{C}$ is considered as a rapid process. 


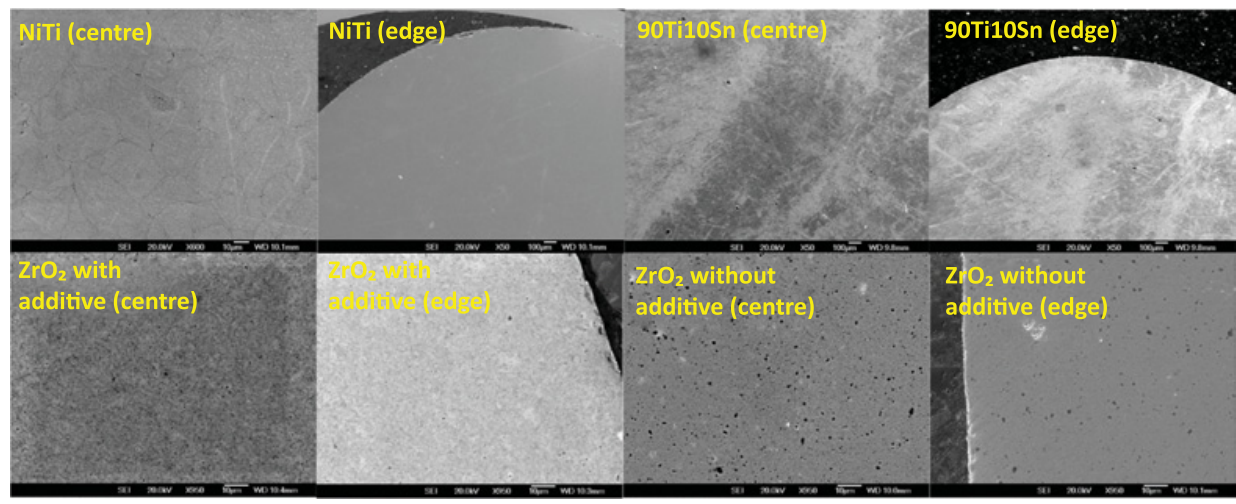

Figure 5. Microstructures of sintered components under SEM.

\section{Analysis of results}

The recorded process graphs and formed samples were examined carefully using the following methods: morphological measurement of sample geometry, relative density calculation by the Archimedean method, and microstructural observation under SEM. All types of powders have been successfully formed into solid cylindrical components, employing a relatively low temperature and sintering period. The results demonstrate that metal powders are very promising materials for the Micro-FAST forming process. With the optimised parameters, all the metal components were of nearly exact $\emptyset 4 \times 4 \mathrm{~mm}$ dimensions. The highest relative density of $99.89 \%$ has been achieved. In addition, even with the high melting point and low electrical conductivity, all of the ceramic parts could be formed with relative density of $95 \%$ or above. It is recognised that the ceramic powders sintering process can be further improved by optimising the process parameters, such as by increasing the sintering temperature and holding time.

\subsection{Samples microstructures}

A group of typical images are selected to show the micro-structure of the samples sintered from different materials under SEM and can be seen in Fig. 5. The relative density for those powders are NiTi-99.89\%, 90Ti10Sn-98.49\%, $\mathrm{ZrO}_{2}$ with additive-95.45\% and without additive-97.53\%. On the polished surface, particle deformation and inter-particle necking can be observed. The edge of each part is smooth and uniformed. Because of the short sintering period, there was no coarsening or grain growth. The samples made from metal powders shows high density with very less residual pores, while from ceramic powders it shows several visible residual pores. However, the residual pores are small and uniformly distributed. No obvious large pores were observed. It is also noticed that, based on the particle size range applied in these experiments, in general, a smaller particle size can achieve a greater relative density than a larger particle size, where a particle size of between 5-20 micros is preferred.

\subsection{Particles' deformation-behaviours}

The particles' deformation can be seen in Fig. 6. AISI420 and NiTi samples are shown as an example, the four particles' (A, B, C and D) boundary relay on each other perfectly after plastic deformation, which results in a good densification. 


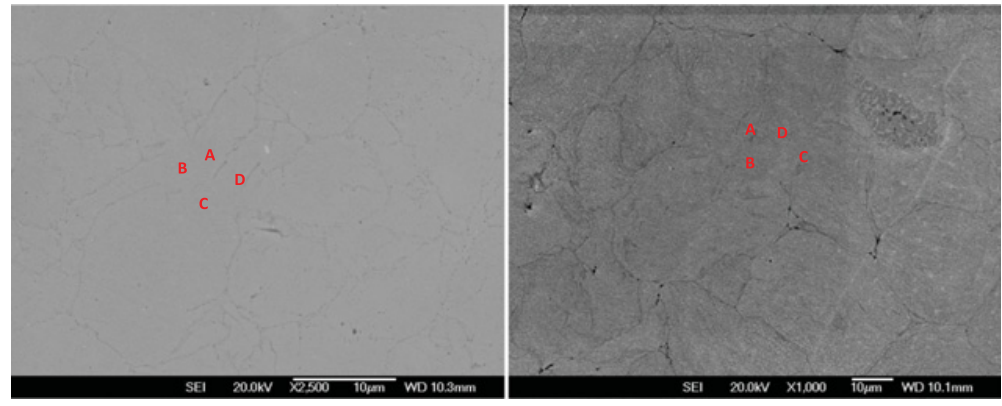

Figure 6. SEM micrographs of formed sample AISI420 (left) and NiTi (right).

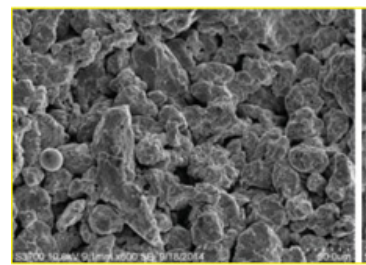

(a)

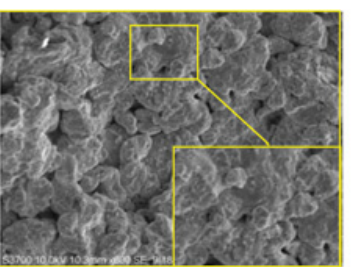

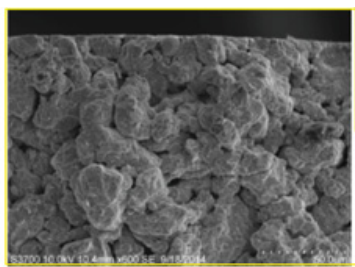

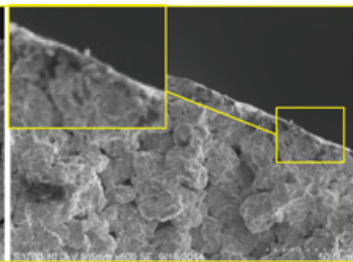

(b)

Figure 7. Microstructures of Titanium alloy under different sintering temperature (a) and forming pressure (b).

\subsection{Influential factors}

High current and low voltage were employed in the Micro-FAST process. When massive AC current passes through the powders and dies, the temperature rises rapidly according to the Joule-Lenz's Law (see Eq. (2)), where the overall heat generated Q is determined by I, the current passing through; R, the resistance; and t, the time. The current applied for the Micro-FAST process ranges from 3-30 kA. The high particle contact resistance also make a great contribute to the fast heating.

$$
Q=I^{2} R t
$$

From the analysis of the formed parts, increases of the heating rate and a higher sintering temperature have a significant influence on the final result. As shown in Fig. 7(a) as an example, by keeping other parameters the same, the Titanium alloy that was sintered at $700{ }^{\circ} \mathrm{C}$ has necking between particles and many residual pores exist. However, when the temperate was increased to $900^{\circ} \mathrm{C}$, only a few residual pores remain, and particle deformation and interfacial melting lead to particles joining together and therefore, to increase in the part density.

Generally, by pressing the powders while sintering, higher densification can be achieved at the similar temperature. It has been verified from these experiments, that the component's quality increases significantly with the increase of the pressure applied. As shown in Fig. 7(b) as an example, by keeping other parameters the same, increase of the forming pressure also leads to an improvement in densification.

\section{Conclusions}

This paper demonstrated that Micro-FAST is a promising process for the rapid forming of microcomponents with a wide range of materials, especially with difficult-to-cut and difficult-to-form 


\section{ICNFT 2015}

materials. The sintering time can be shortened with the optimised sintering parameters. Ceramic materials can be sintered successfully at a relatively low sintering temperature and sintering time. Future work will be focussed on the investigation of influential sintering parameters in order to optimise the process and to increase its repeatability, to ensure greater quality of the parts to be produced.

The authors would like to acknowledge the funding support from European Commission through FP7 FOF MicroFAST Project (GA No. 608720)). The authors would specially thank Prof. Jianguo Lin and his colleagues in Imperial College London for providing the Gleeble machine for conducting the experiment and for their kind support through the experiment. Authors would also like to thank EU Micro-FAST project partners who provide powder materials for conducting the experiments.

\section{References}

[1] Y. Qin, A. Brockett, Y. Ma, A. Razali, J. Zhao, CS. Harrison, W. Pan, X. Dai, \& D. Loziak, The International Journal of Advanced Manufacturing Technology, 47, 9-12, pp. 821-837 (2010)

[2] S. Grasso, Y. Sakka and G. Maizza, Science and Technology of Advanced Materials, 10, 5 (2009)

[3] A.G. Bloxam, GB patent 27002 (1906)

[4] R. Orrù, R. Licheri, A. Locci, A. Cincotti, G. Cao, Materials Science and Engineering, 63, 4-6, pp. 127-287 (2009)

[5] K. Huang, et al., Materials and Manufacturing Processes, 28, 2, pp. 183-188 (2013)

[6] D. Lu, et al., Journal of Microelectromechanical Systems, 22, 3, pp. 708-715 (2013) 